\title{
Energy dependence of the Nernst-Ettingshausen effect induced by pulsed laser light in bismuth films
}

\author{
M. Sánchez Balmaseda \\ Departamento de Física Aplicada III (Electricidad y Electrónica), Facultad de Ciencias Físicas, \\ Universidad Complutense, 28040 Madrid, Spain \\ J. C. G. de Sande and J. M. Guerra Pérez \\ Departamento de Optica, Facultad de Ciencias Fisicas, Universidad Complutense, 28040 Madrid, Spain
}

(Received 26 July 1994)

\begin{abstract}
The behavior with the irradiation energy and with the magnetic field of the thermomagnetic response induced by laser pulses in $5.5-\mu \mathrm{m}$ Bi films at room temperature is reported in this paper. The Nernst-Ettingshausen coefficient at an applied magnetic field of $1 \mathrm{~T}$ is estimated: $Q_{\mathrm{NE}} \simeq 1.0 \times$ $10^{-5} \mathrm{~V} / \mathrm{T} \mathrm{K}$. A good agreement is found when these results are compared with those reported earlier in polycrystalline bulk samples obtained by a conventional method. This supports the reliability of the pulsed laser technique in the measurement of weak transport effects and indicates that the optically pumped carriers hardly influence the transport properties of bismuth.
\end{abstract}

\section{INTRODUCTION}

Laser-induced phenomena in conductive films have been the subject of study for several years. Part of the interest in this type of experiment is its possible application in the development of optical detectors. ${ }^{1,2}$ On the other hand, important information about materials properties can be deduced, although not always in a simple or direct form. Since experiments are usually carried out with pulsed lasers, obtaining materials properties requires not only the use of physical models to explain the material behavior, but the use of numerical and mathematical techniques as well. In compensation, the pulsed laser excitation has some experimental advantages. Very high temperature gradients can be produced in thin films with a moderate change in the average temperature of the sample. ${ }^{3,4}$ In the case of weak thermoelectric or thermomagnetic effects, a big thermal gradient improves the sensitivity of the method. On the other hand, continuous wave laser excitation produces low thermal gradients and frequently an appreciable temperature change. Also, pulsed excitation can induce interesting dynamical phenomena related to basic materials features. In previous publications we have reported the anomalous response of bismuth films to irradiation with $1.064-\mu \mathrm{m}$ laser pulses with and without an applied magnetic field along the film. The temporal evolution of both thermomagnetic and thermoelectric responses did not follow the temporal evolution of the foreseeable thermal gradient. Experimental results were well explained if an optical pumping of carriers into a metastable conduction band was considered. The recombination of the excited carriers resulted in a delayed heat source, which explained the abnormally long decay time of the induced thermal gradient. ${ }^{5,6}$ Recently, a photoinduced conductivity change in bismuth films has been observed as well. ${ }^{7}$ Some additional effects may be expected due to the presence of these metastable carriers. Thus, the thermoelectric and thermomagnetic coefficients could be modified.

When a film is irradiated with a laser pulse and a magnetic field $\mathbf{B}$ is simultaneously applied, an electrical field $\mathbf{E}_{\mathrm{NE}}$ is generated which is proportional to the thermal gradient $\nabla T$ induced by the laser pulse in the form

$$
\mathbf{E}_{\mathrm{NE}}=Q_{\mathrm{NE}} \mathbf{B} \times \nabla T
$$

where $Q_{\mathrm{NE}}$ is the Nernst-Ettingshausen coefficient.

This work reports the measurement of the thermomagnetic response of $\mathrm{Bi}$ films at different magnetic fields to 1.064- $\mu \mathrm{m}$ laser pulses when the irradiation energy is varied. From the experimental data, an estimation of the Nernst-Ettingshausen coefficient is obtained.

\section{EXPERIMENTAL PROCEDURE}

\section{A. Sample preparation and characterization}

Films were grown on glass substrates by vacuum evaporation ( $10^{-6}$ Torr) of $99.9999 \%$-pure Bi. Before evaporation the substrates underwent a standard process of ultrasound cleaning. The film thickness was controlled by a quartz crystal monitor during evaporation and later confirmed by a surface-profile analyzer. Samples of 5.5 $\mu \mathrm{m}$ were evaporated at room temperature $(300 \mathrm{~K})$. The films's configuration on the glass substrate, as well as the design of the support's connections to extract the electrical signal, are shown in Fig. 1. The films were circular, having a diameter of $4.2 \mathrm{~mm}$. The dc electrical resistance of the samples was about $1.2 \Omega$.

Films grown under identical conditions, having thicknesses between 80 and $90 \mathrm{~nm}$, were analyzed by electron- 


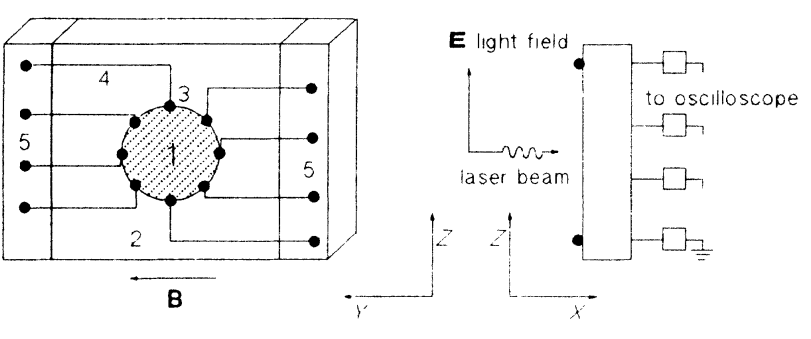

FIG. 1. Sample design and field geometry: 1, Bi film;2, glass substrate; $3, \mathrm{Ag}$ paste; 4 , Cu electrical contacts; 5 , isolator.

transmission microscopy. The films had a microcrystalline structure, the trigonal axis being perpendicular to the substrate and the binary axis being randomly oriented. The grain size was of the same order of magnitude as the film thickness.

\section{B. Measurement system}

The films were irradiated with a $\mathrm{Q}$-switched and polarized Nd-YAG laser (wavelength of $1.064 \mu \mathrm{m}$ ). A typical impinging laser pulse had a temporal width of $\simeq 5 \mathrm{~ns}$ and an energy of $\simeq 6 \mathrm{~mJ}$ evenly distributed on $\mathrm{a} \simeq 15.2$ $\mathrm{mm}^{2}$ section where the sample was placed. The energy was attenuated by an adequate number of neutral filters to obtain irradiation energy densities between 2 and 70 $\mathrm{J} / \mathrm{m}^{2}$.

The samples were placed between the polar pieces of an electromagnet and held by a positioner which allowed for perpendicular adjustment between the laser beam and the film, and parallel adjustment between the magnetic field and the film. The light polarization plane was perpendicular to the magnetic field (see Fig. 1).

A beam splitter was used to divide the laser beam. The first of these beams was detected by a $<1$ ns rise time photodiode to be used as a monitorizing signal. This first beam was also used to synchronize a transient programmable digitizer. The second beam fell on the surface of the film. The electrical potentials generated in the film were registered in the transient programmable digitizer, its 3-dB bandwidth being $400 \mathrm{MHz}$. After having synchronized the oscilloscope trigger, the first signal was registered with a preestablished delay in the same scan as the pulse generated in the film. The sample was directly linked with the oscilloscope through a BNC connector. The electrical lines were impedance matched at $50 \Omega$. All measurements were performed at room temperature.

\section{RESULTS}

In a previous work we studied the transverse thermoelectric potential that is generated in bismuth films when they are irradiated with infrared laser pulses. The contacts design of the sample allowed us to measure the thermoelectric response of the film in four different directions and the measurements showed a clear Lambertian dependence of this voltage. In the present work, measurements

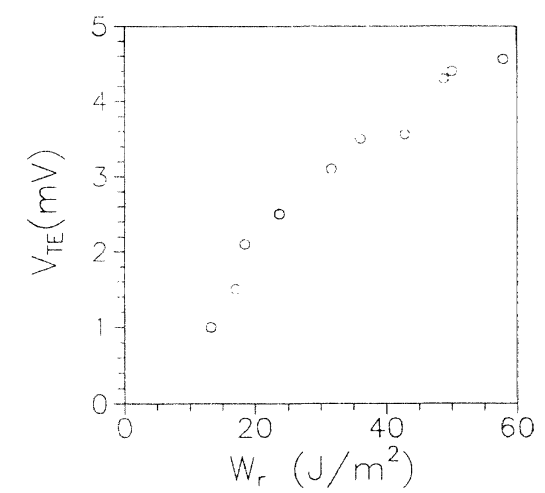

FIG. 2. Dependence of the peak value of the thermoelectric potential $V_{\mathrm{TE}}$ on the irradiation energy density.

were performed in the direction of the lowest thermoelectric response where this signal was not measurable for low irradiation energies. Since the thermoelectric potential increases as the irradiation energy increases, at high energies the thermoelectric effect was more significant. Figure 2 shows the thermoelectrical response of the film for energies between 10 and $60 \mathrm{~J} / \mathrm{m}^{2}$. In order to measure a possible dependence of this thermoelectric potential on the magnetic field, the photoinduced signal was measured in the direction of maximum thermoelectric response under a magnetic field applied as indicated in Fig. 1 and ranging from 0 to $1 \mathrm{~T}$. Inverting the direction of the magnetic field, another sequence of readings was taken. Thus, if with the first geometry the thermomagnetic and the thermoelectric signals had the same sign, with the second geometry those signals had opposite signs. This permitted us to isolate the thermoelectric potential, which did not show any measurable dependence on the magnetic field.

The pulses generated in the films were studied under different irradiation energies at two fixed magnetic fields of 0.6 and $1 \mathrm{~T}$. Readings were taken of the peak of the irradiation pulse $V_{1}$ and that of the induced pulse. The peak of the thermomagnetic potential $V_{2}$ was obtained by subtracting the peak value of the thermoelectric potential from the registered maximum for each energy. The ratio $V_{2} / V_{1} B$ would, in principle, represent a quantity proportional to $Q_{\mathrm{NE}}$. Figure 3 shows a positive $Q_{\mathrm{NE}}$ that

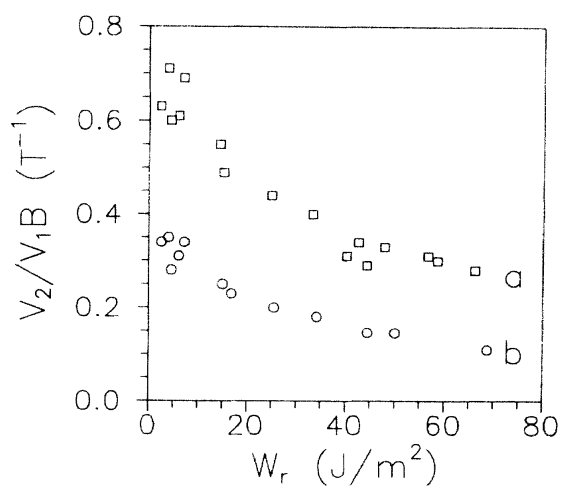

FIG. 3. Dependence of $V_{2} / V_{1} B$ on the irradiation energy density for applied magnetic fields of $1 \mathrm{~T}$ (curve $a$ ) and $0.6 \mathrm{~T}$ (curve $b$ ). 


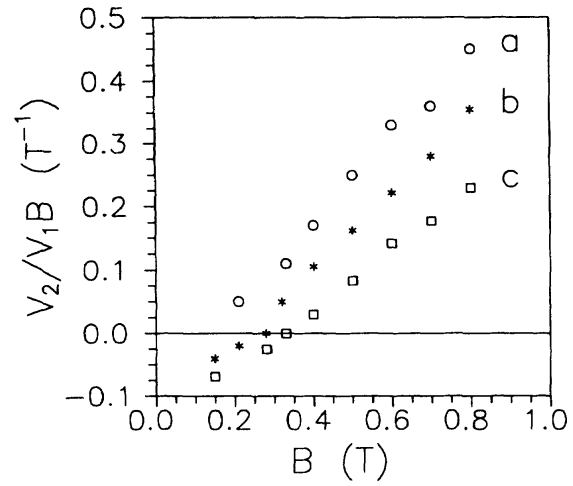

FIG. 4. Dependence of $V_{2} / V_{1} B$ on the magnetic field for irradiation energy densities of $w_{r} \simeq 10 \mathrm{~J} / \mathrm{m}^{2}$ (curve $\left.a\right), w_{r} \simeq 21$ $\mathrm{J} / \mathrm{m}^{2}$ (curve $b$ ), and $w_{r} \simeq 50 \mathrm{~J} / \mathrm{m}^{2}$ (curve $c$ ).

decreases rapidly as the irradiation energy increases, the decreasing being slower for energies higher than $40 \mathrm{~J} / \mathrm{m}^{2}$.

The film response when the applied field is varied from 0.15 to $0.8 \mathrm{~T}$ at a fixed energy is showed in Fig. 4. Curves $a, b$, and $c$ give the results for irradiation energies of 10 , 21 , and $50 \mathrm{~J} / \mathrm{m}^{2}$, respectively. Curve $a$ shows a positive coefficient with a monotonous increasing behavior already observed. ${ }^{8}$ At higher energies the response presents a change in sign, being the generated potential negative at low magnetic fields (curves $b$ and $c$ ).

The experimental points in all figures correspond to an average of 10 registers.

\section{DISCUSSION AND CONCLUSIONS}

The Boltzmann equation in the relaxation time approximation predicts a decreasing behavior of the absolute value of the coefficient $Q_{\mathrm{NE}}$ as the carrier density increases when only one kind of carrier and one type of scattering process are assumed. This equation under the same assumption gives a decreasing behavior of that coefficient with the magnetic field. ${ }^{9,10}$ However, when more than one type of carrier with different scattering mechanisms is considered in the resolution of the problem, a more complicated behavior of $Q_{\mathrm{NE}}$ is found.

From our experimental data, the Nernst-Ettingshausen coefficient $Q_{\mathrm{NE}}$ can be calculated in the form

$$
Q_{\mathrm{NE}}=\left(1+\frac{R_{i}}{R_{o}}\right) \frac{V_{2}}{l B \nabla T_{p}},
$$

where $R_{i}$ is the internal resistance of the sample, $R_{o}$ is the oscilloscope input impedance $(50 \Omega), l$ is the distance between electrodes that coincides with the illuminated length, and $\nabla T_{p}$ is the peak value of the temporal profile of thermal gradient which has been spatially averaged along the thickness. The thermal gradient is calculated making use of the heat-diffusion equation

$$
\rho^{*} c_{p} \frac{\partial T(x, t)}{\partial t}=\phi_{s}(x, t)+K_{t} \frac{\partial^{2} T(x, t)}{\partial x^{2}},
$$

where $T(x, t)$ is the temperature at a given distance $x$ from the film surface and at a given instant $t, \rho^{*}$ is the mass density, $c_{p}$ is the heat capacity, $K_{t}$ is the thermal conductivity, and $\phi_{s}(x, t)$ is the source term. The laser power is mainly absorbed through interband transitions and the excited electrons transmit part of their energy to the lattice in a quick nonradiative relaxation process occupying the levels that are closest to the band edge. The rest of the energy is liberated in a recombination process that takes a time of about some tens of nanoseconds. A good estimation of the induced thermal gradient can be obtained if we assume that excited carriers remain frozen in the position where they were pumped by the laser light. ${ }^{5,6}$ In this case, the source term is the following:

where

$$
\begin{aligned}
\phi_{s}(x, t)= & \left(1-\frac{E_{m}}{h \nu_{L}}\right) \phi_{0} \exp (-\delta x / d) f(t) \\
& +E_{m} S_{m} n(x, t),
\end{aligned}
$$

$$
\phi_{0}=\frac{A w_{r}}{\int_{0}^{d} \exp (-\delta x / d) d x \int_{0}^{\infty} f\left(t^{\prime}\right) d t^{\prime}},
$$

and $n(x, t)$ is the density of pumped pairs given by

$$
\begin{aligned}
n(x, t)= & \frac{\phi_{0} \exp (-\delta x / d)}{h \nu_{L}} \\
& \times \int_{0}^{t} f\left(t^{\prime}\right) \exp \left[-\left(t-t^{\prime}\right) S_{m}\right] d t^{\prime},
\end{aligned}
$$

where $E_{m}$ is the average recombination energy, $h \nu_{L}$ is the photon energy, $\delta=d / x_{L}, x_{L}$ is the attenuation distance of the laser radiation in the material, $d$ is the film thickness, $f(t)$ is the temporal profile of the laser pulse, $S_{m}$ is the excited carrier recombination rate, $A$ is the absorption coefficient, and $w_{r}$ is the ratio $E_{r} / s$, with $E_{r}$ the laser pulse energy and $s$ the beam cross section at the film position.

In order to avoid an appreciable temperature rise of the film surface and a big dispersion in the experimental data, an energy density of $w_{r} \simeq 15 \mathrm{~J} / \mathrm{m}^{2}$ is chosen. Making use of the laser pulse register $f(t)$, with the experimental data $d \simeq 5.5 \mu \mathrm{m}$ and $h \nu_{L} \simeq 1.17 \mathrm{eV}$, and taking the previously estimated values $E_{m} \simeq 0.64 \mathrm{eV}, S_{m}^{-1} \simeq 57$ $\mathrm{ns},{ }^{5-7}$ the temporal evolutions of the film temperature and of the generated thermal gradient are obtained. The bismuth physical constants $A=0.33, x_{L}=20 \mathrm{~nm}, c_{p}=$ $125.4 \mathrm{~J} / \mathrm{kgK}, \rho^{*}=9747 \mathrm{~kg} / \mathrm{m}^{3}$, and $K_{t}=6.0 \mathrm{~W} / \mathrm{mK}$ are taken from current literature. ${ }^{11,12}$ This calculation gives a spatially averaged temperature increase below $1 \mathrm{~K}$ and a peak value of the thermal gradient $\nabla T_{p} \simeq 1.3 \times 10^{6}$ $\mathrm{K} / \mathrm{m}$. The highest increase of the surface temperature is about $7 \mathrm{~K}$. If we neglect the effect of this temperature change, Eq. (2) gives an estimation of the thermomagnetic coefficient at $B \simeq 1 \mathrm{~T}$ and at room temperature: $Q_{\mathrm{NE}} \simeq 1.0 \times 10^{-5} \mathrm{~V} / \mathrm{TK}$, where we have used the experimental values $V_{2} \simeq 52 \mathrm{mV}$ and $l \simeq 4.2 \mathrm{~mm}$. This estimated value is in very good agreement with the one measured in polycrystalline bulk samples of pure bismuth at $275 \mathrm{~K}$ and at $B=1 \mathrm{~T}$ by means of a conventional method: $Q_{\mathrm{NE}} \simeq 1.2 \times 10^{-5} \mathrm{~V} / \mathrm{TK}^{10}$ Moreover, the dependences of both measured coefficients on the magnetic 
field are very similar. This coincidence seems to point out that pumped carriers have a very small influence on the coefficient $Q_{\mathrm{NE}}$ and that the pulsed laser technique is useful to obtain quantitative and accurate information of transport coefficients.

Considering the energy density $w_{r} \simeq 15 \mathrm{~J} / \mathrm{m}^{2}$, the maximum of the spatial average of $n(x, t)$ given by Eq. (6) is $n \simeq 3.8 \times 10^{24} \mathrm{~m}^{-3}$. This density of excited carriers is somewhat higher than the intrinsic carrier density at room temperature $n_{i} \simeq 2.9 \times 10^{24} \mathrm{~m}^{-3} .^{10}$ If metastable carriers had transport properties similar to those of intrinsic carriers, the coefficient $Q_{\mathrm{NE}}$ would be positive and monotonously decreasing with the magnetic field, behavior absolutely different from the one here reported. If we did not consider an optical pumping of carriers into a metastable band in the calculation, the expected temperature gradient would be much higher, that is, a smaller value for $Q_{\mathrm{NE}}$ would be found.

All these considerations mean that pumped carriers influence mainly the temperature gradient value but do not play an important role in the induced transport phenomenon. This result agrees with our recent estimations that yield low diffusion coefficients and low mobilities for carriers in the metastable band. ${ }^{7}$

The only experimental data of the behavior of $Q_{\mathrm{NE}}$ versus temperature near room temperature correspond to $\mathrm{Bi}$ single crystals, showing a decreasing behavior of the absolute value of the thermomagnetic coefficient as temperature increases. Nevertheless, the dependence of $Q_{\mathrm{NE}}$ on the magnetic field is very different in these samples from the one reported here. ${ }^{13}$

Taking into account the above discussion, it seems that the observed behavior of the coefficient $Q_{\mathrm{NE}}$ with the irradiation energy would be mainly due to a lattice temperature increase which is mostly produced near the film surface and that the metastable carriers have no significant influence on the thermomagnetic coefficient.
${ }^{1}$ H. Lengfellner, S. Zeuner, W. Prettl, and K. F. Renk, Europhys. Lett. 25, 375 (1994).

${ }^{2}$ S. M. Javed Akhtar and D. Ristau, Phys. Status Solidi A 109, 255 (1988).

${ }^{3}$ H. E. Elsayed-Ali and J. W. Herman, Appl. Phys. Lett. 57, 1508 (1990).

${ }^{4}$ S. S. Mann, B. D. Todd, J. T. Stuckless, T. Seto, and D.

A. King, Chem. Phys. Lett. 183, 529 (1991).

${ }^{5}$ M. Sánchez Balmaseda and J. M. Guerra, Phys. Rev. B 41, $10372(1990)$.

${ }^{6}$ J. C. G. de Sande, M. Sánchez Balmaseda, and J. M. Guerra, Phys. Rev. B 47, 9844 (1993).

${ }^{7}$ J. C. G. de Sande, M. Sánchez Balmaseda, and J. M. Guerra, Phys. Rev. B 50, 1861 (1994).
${ }^{8}$ M. Sánchez Balmaseda and J. M. Guerra, Phys. Rev. B 40, 8252 (1989).

${ }^{9}$ B. M. Askerov, B. I. Kuliev, and S. R. Figarova, Phys. Status Solidi B 121, 11 (1984).

${ }^{10}$ J. C. G. de Sande and J. M. Guerra, Phys. Rev. B 45, 11469 (1992).

${ }^{11}$ AIP Handbook, 3rd ed., edited by Dwight E. Gray (AIP, New York, 1972).

${ }^{12}$ Handbook of Chemistry and Physics, 53rd ed., edited by Robert C. Weast (Chemical Rubber Co., Cleveland, OH, 1972).

${ }^{13}$ J. P. Michenaud, E. Cheruvier, and J. P. Issi, Solid State Commun. 9, 1433 (1971). 